\title{
RECENT ADVANCES IN THE PRODUCTION AND APPLICATION OF X-RAYS.*
}

BY

\author{
J. S. SHEARER, B.S., PH.D. \\ Professor of Physics, Cornell Liniversity.
}

There have been from time to time during the century past some remarkable discoveries destined to cause profound changes in human thought and mode of life. Some of these have become so quickly and firmly incorporated inl our environment that we have hardly realized their origin and rapid development. In theoretical and applied electricity are some of the most striking examples of such developments, having wrought such wonderful results that one can hardly realize their ramifications.

Measured in terns of the duration of human history, all electrical knowledge is reccnt and most of the practical applications were made within the memory of those who are near to man's allotted three score years and ten. In Denmark there has just been celebrated the one hundredth anniversary of Oersted's discovery of the relationship between electric and magnetic phenomena. A few years after Oersted's work, Henry and Faraday laid the foundation for the marvels to be accomplished in applied electricity; Less than fiity years ago the first power motor made in America was completed and one of the designers is still living. Many of the pioneers in the development of the arc and the incandescent lamp and of the telephone are still active.

'Twenty-five years ago this month there was announced a new wonder, the culmination of a long series of experiments, the starting point of a new line of endeavor, giving rise to concepts, unrivalled in scope and daring, and, curiously enough, resulting in theories strangely related to those of the master mind whose honored name is borne by this society.

A careful and well-trained observer, already well known in his chosen field, Röntgen not only announced a type of radiation whose existence had not been suspected but he described, with

* Presented at a meeting of the Section of Physics and Chemistry held Thursday, December 9, ID20.

VOL. 192, NO. II5I-43 
wondrous simplicity and clearness, experiments performed to ascertain its nature and to properly place it in the scheme of physical science.

I well remember in Dr. E. L. Nichols' physics seminary the mention of a rumor that a radiation had been found alle to pass through opaque objects, not capable of refraction or reflection and otherwise strange in its nature. Curiously enough it did not strike us as at all peculiar that light should penetrate glass or water or that radiant heat shouid pass through opaque hard rubber, but the announcement of rays that would pass through aluminum or the human body was received with hardly tolerant skepticism.

Briefly stated Röntgen showed that peculiar rays were produced by electric discharge through a highly exhausted tube. They were able to excite fluorescent light in certain materials and to affect a photographic plate. They were not refracted or reflected, were propogated in straight lines, and were able to produce secondary rays possessing like properties. Materials varied in transparency to this radiation somewhat in the order of their densities. In his first announcement he was not certain as to the point of origin of the rays or the basic mechanism of their production. He used an induction coil and, initially, tubes that had been designed to demonstrate previously known phenomena. In fact the presence of a card covered with crystals often used in cathode ray tubes led to the discovery.

In a comparatively short time it became clear that the rays originated mainly, at least, at the surfaces of objects within the tubes or in the glass walls, and very soon the curved cathode of Crookes and the metal target gave us the "focus" tube. These early tubes were operated either by induction coils or by "static" machines and carried very little electric energy. The demand for rapid radiography resulted in the development of more powerful coils and the slow evolution of tubes with massive metal targets, water cooling, etc., to enable more rapid work. Meanwhile but little advance had been made in basic theory or in applications outside of medicine and surgery.

Let us consider first the evolution of the exciting apparatus. The early high tension generators had certain inherent complications and limitations. The static machine gave an almost ideal discharge as to steadiness but of very limited quantity. 
Also, it was often difficult to get it to function. The induction coil required an interrupter to break a direct current and a properly proportioned condenser to get full output. The wave torm might be very irregular as may be seen by oscillograms. There was always more or less "inverse," a source of serious trouble in ail tube operation.

FIG. I.

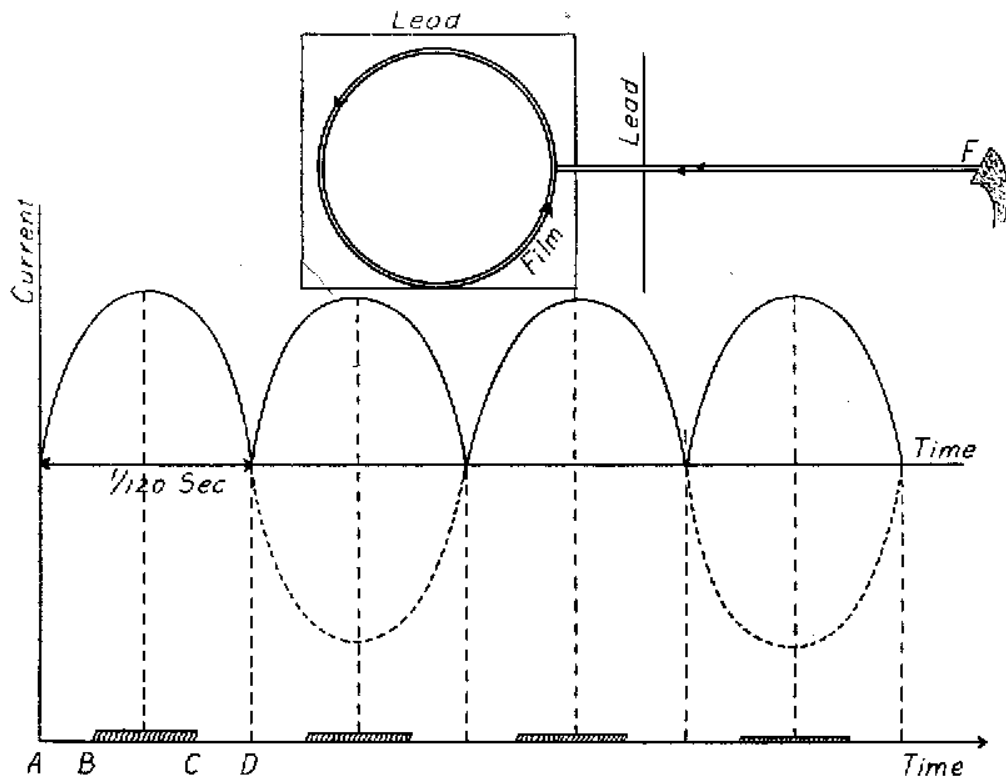

$F$ targel of $X$-ray tube. A narrow beam of rays passes the lead slits and reaches the film on the revolving cylinder. When a synchronous rectifier is used we have four half waves per revolution as shown by the continuous line.

At the instants marked $A D$, etc., there is no tube current and no X-rays. During the time $A B$ and again $C D$ there is tube current but the voltage is too low to give effective radiation.

When no synchronously driven switch is used the half waves shown as dotted lines are not abie to pass through the tube.

The X-ray transformer, developed to operate on alternating current, required a strictly synchronous motor to operate a revolving switch. Such devices were relatively complicated, bulky and licavy and were constructed to deliver enormonsly more power than could be utilized in practice. Thus a tube may operate on a power intake of $3 \mathrm{k}$. W., but machines of ro to $\mathrm{I} 5 \mathrm{k}$. W. contimuous rating were supplied, although only a single tube was to be operated at one time. Now the output of an X-ray tube is dependent on the current through it and on the voltage drop 
between its terminals. The voltage is a much more important factor than the current as the radiation at a given current increases at least in proportion to the square of the voltage. During a single set of current changes from no current through all its variation back to no current again there will be, at each instant, some voltage and current, but only when the voltage is high enough

Fig. 2 .

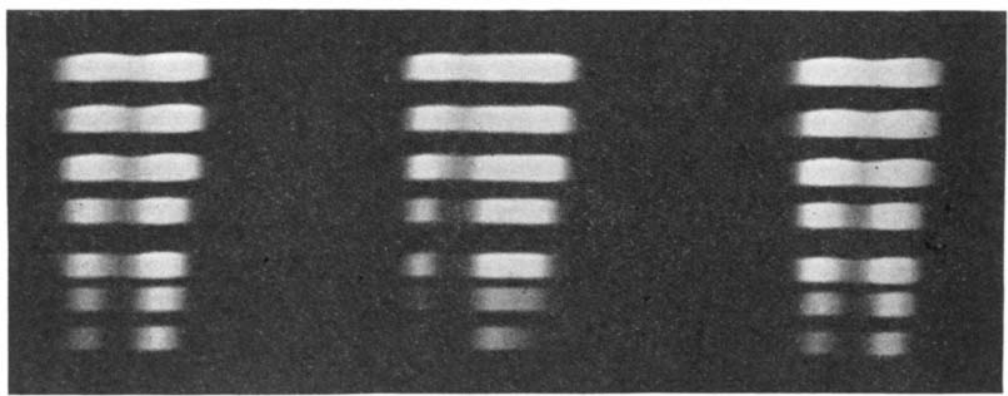

A series of cxposures using the apparatus of Fig. I. One cxposure has been cut out to shorten the figure. Four exposures result from one revolution ol the motor.

Note the clear spaces between the dark strips showing that current at low voltagc is ineffective. The longest strips were madc at $45 \mathrm{k}$. v. the others at 40 , 35 . etc., to $\mathrm{I}_{5} \mathrm{k}$. v. An oscillation caused a voltage drop distorting the wave from giving the light areas in the exposed strips.

FIi. 3.

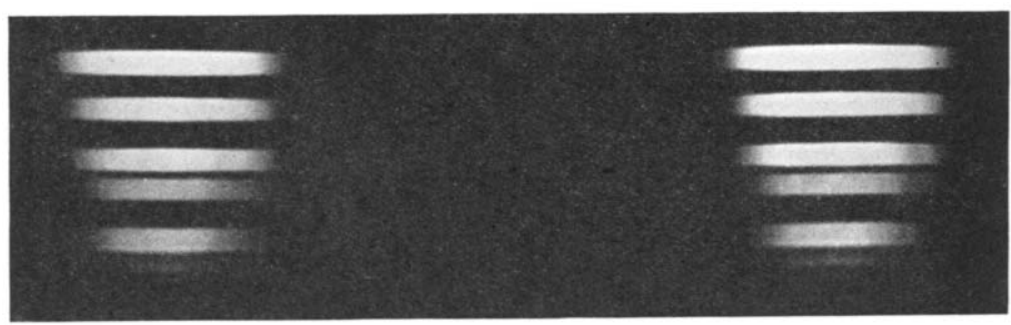

Shows a similar set using a hot cathode tube, self-rectifying. Only two spots per revolution.

for the production of rays able to pass out through the walls of the tube will we get a delivery of $\mathrm{X}$-rays. This is shown by photographing the $X$-ray delivery on a moving film attached to a cylinder rotated by a synchronous motor and operated on the same circuit as the $\mathrm{X}$-ray transformer. The rays pass through a narrow slit and each area in the photographs shown was exposed 450 times ( 5 sec.). Note the much greater in- 
tensities at the higher voltage all other factors, aiz., time, current, distance and development being the same. Note also those of a self-rectifying tube, where only two spots appear per revolution.

When we introduce aluminum in the path we find much of the radiation sent out during low voltage fails to pass through

FIG. 4.

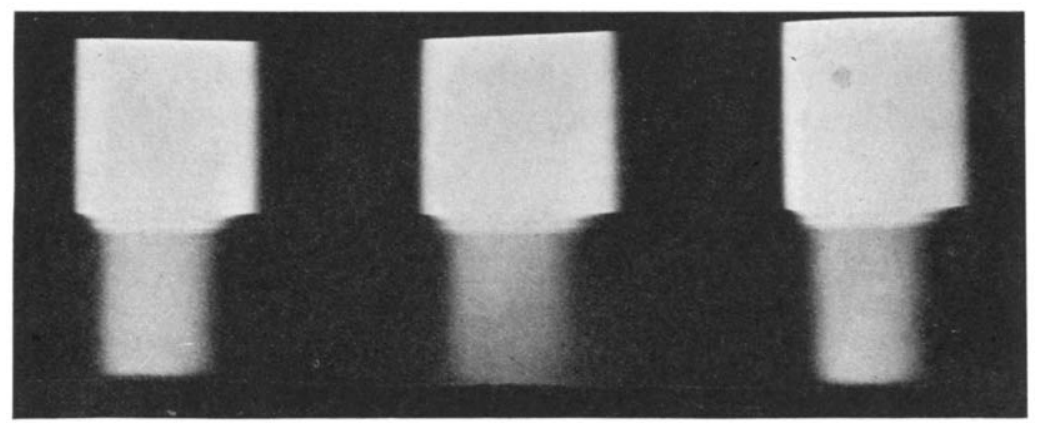

One half of the slit of Fig. I was covered by $4 \mathrm{~mm}$. of aluminum. On a $6^{\prime \prime}$ gap only $1 / 4$ the time was given to the upper portion in order to nearly equalize the exposures. More of the radiation at the higher gap is able to pass through the aluminum.

FIG. 5.

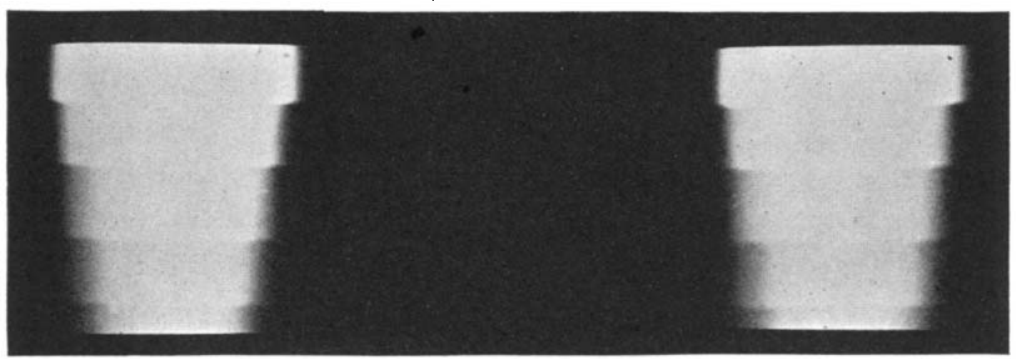

Reduction by absorption of several millimeters of aluminumı, o, I, 2, 3, and $4 \mathrm{~mm}$. filters used.

even a thin layer. Clearly the most efficient use of electric energy, i.e., the greatest $\mathrm{X}$-ray delivery for a given heat produced at the target would be when all the current is passed at the same potential, i.e., when the voltage is unfluctuating. The work of Hull showed that $2 \mathrm{M}$. A. at a constant voltage produced rarliation identical in all respects to that given by $3 \mathrm{M}$. A. sine wave. Appliances to give unfluctuating voltages of roo $\mathrm{k}$. $\mathrm{v}$. or more are 
expensive and will probably not be generally used in practice, but will be of enormous value in research and standardization. And it is not at all unlikely that simpler methods may be found to secure at once the highest voltage and a steady output.

Eighteen years after Röntgen's discovery, or in $19 \mathrm{I} 3$, began what I would designate as the recent period, due to two remarkable discoveries or deveiopments. One the hot cathode tube by Coolidge, the other diffraction of $\mathrm{X}$-rays and resulting interference phenomena by Laue. The former gave us a tube of stable and reproducible performance, the other not only indicated the nature of X-rays but has proved a powerful tool in their application.

Do not conclude that I wish to imply that nothing was accomplished in preparation for these results in the interim between I 895 and I9 13. For, starting with Röntgetn's experiments there followed the discovery of radioactivity ultimately resulting in the electron theory of electricity, or perhays I should say, of matter, and the Coolidge tube may be said to be a nost perfect application of these new developments. Machines for rectifying high-tension alternating currents had been perfected by Snook and others so that high-power exciters to operate the powerful new tube were already in use.

The Coolidge tube when the anode is cold, i.e., below a tew hundred degrees centigrade, permits curtent to pass in only one direction, i.e., is self-rectifying. Early in 1917 Doctor Coolidge undertook to develop a tube primarily for army use, utilizing this principle and eliminating the synchronously driven switch. In order to carry away the heat developed at the anode he returned to the use of a tungsten button set in copper and utilized an outside radiator also previously employed with gas tubes. This tube was soon known as the radiator type Coolidge, and was used in the $\mathrm{C}$. S. A. portable and bedside outfits. Although limited to $10 \mathrm{H} . A$ at $60 \mathrm{k} . \vee$, or $.6 \mathrm{k}$. w., its performance marked a new era in medical röntgenology. These tubes are now furnished for $30 \mathrm{M} . \mathrm{A}$, and on account of small size and the simplicity of operation are likely to largely displace the "interrupterless transformer" except for therapy. What the future holds for this field one hardly dares to predict, but surely the end is not yet.

Let us now summarize the main features in the production 
and the known propertics of X-rays as a basis for the consideration of their applications.

I. They are due to the sudden change in velocity of electrons, i.e., small natural negative charges.

2. They travel in straight lines from the points where the electrons are stopped.

3. Their velocity is probably the same as that of light.

4. They are reflected by atoms in proportion to the atomic weight of the atoms struck.

5. They excite relatively long wave-length radiation known as fluorescent light in a few materials.

6. They tear electrons from atoms in their flight, rendering gases conducting by ionization, and causing photoelectric effects in liquids and solids.

7. They affect photographic emulsions in a manner similar to light.

8. They show diffraction spectra analogous to light when a sufficiently fine atomic lattice is used as a grating.

9. The X-ray spectrum depends on the voltage of tube operation and on the target material. No tube giving homogeneous rays, i.e. of a single wave-length, is possible.

I0. The atomic reflection in a body whose atcms are not organized into crystalline structure resembles the scattering of light by fog. That of a crystal is similar to crossed grating spectra.

II. There are discontinuous spectra as well as continuous as is the case with light sources. The bright line visible spectrum of the incandescent gas is shown at sufficiently high tomperature while the characteristic lines of the target material are only shown in superposition on the continuous spectrum when we use a voltage above a minimum, characteristic of each target material.

12. The distribution of radiation among various wave-lengths and the total amount of radiation is profoutndly modified by the voltage used which determines the electron speed. Voltage is here analogous to temperature in black body radiation.

13. The absorption of materials shows discontinuities intimately connected with their characteristic emission spectra. This also is analogous to light, absorption and emission.

14. The minimum wave-length emitted is a function of the maximm voltage available. 
15. The extrenely short waves are less easily absorbed and thus effects produced through thick masses or dense absorbers are due to the shorter waves of the $\mathrm{X}$-ray spectrum.

Aside from their action on living cells, $X$-ray applications are based on (I) the absorption effect of matter varying with atomic weight and thickness, (2) on diffraction effects.

The radiation received on a photographic plate or a fluorescent screen shows shadows, i.e., areas of reduced brightness on the screen or increased transparency on the plate, resulting frotn either a density exceeding that of surrounding material or greater thickness or both. In the varicus applications this rariation of opacity must be interpreted in the light of acquired experience. The general theory is approximately as follows:

If $Q=$ radiation per sq. $\mathrm{cm}$. falling on the proximal surface

$Q_{2}=$ radiation falling on a photographic plate alter passing through a thickness $d$ of homogenous material.

$Q_{1}=$ that having passed a thickness, $\mathrm{d}-\mathrm{h}$, of the above material and $h$ c.m. of a denser material.

Assume, what is only approximately true, that $\mu$ is the absorption coefficient of the main body and $\mu_{1}$ that of dense region, where $\mu_{1}>\mu$.

$$
\begin{aligned}
& Q_{2}=Q_{\varepsilon}-\mu \mathrm{d} \\
& Q_{1}=Q_{\varepsilon}-\mu\left(\mathrm{d}-\mathrm{h}_{1}\right)-\mu_{\mathrm{h}} \mathrm{h} \\
& \mathrm{Q}_{2}-\mathrm{Q}_{\mathrm{I}}=Q_{\varepsilon}-\mu \mathrm{d}\left[1-\mathrm{c}-\left(\mu_{1}-\mu \mathrm{j} \mathrm{h}\right]\right.
\end{aligned}
$$

But $Q_{2}-Q_{1}$ must fix the possible contrast between these two areas. This will increase with $h$, and with the difference in absorption coefficients for the two materials. Since it is also necessary to get some radiation through the body neither $d$ nor $\mu$ can be too large.

We must then use sufficiently short wave-lengths to affect the screen or plate, but not so high that $\mu_{1}$ and $\mu$ will approach one another. Thus, if all rays were so penetrating as to go through flesh and bone equally well there would be no radiograph, also the bodies must not be too thick or $Q_{2}=Q_{1}=0$.

But reference to to above shows another very important property that always works against the simple theory, viz., rays do not all go straight through but each atom takes a minute toll and sends these rays in other directions, we do not know whether 
or not a single atom scatters in a single direction according to its orientation or not, but it is quite immaterial as there are so many in all sorts of positions that there is a general scattering. There results an undershot radiation, falling where otherwise the radiation is more or less reduced and contrast is lost. In only two ways can this action be overcome, first, by reducing the number of atoms causing scattering by using a diaphragm, thus limit-

FIt. 6.

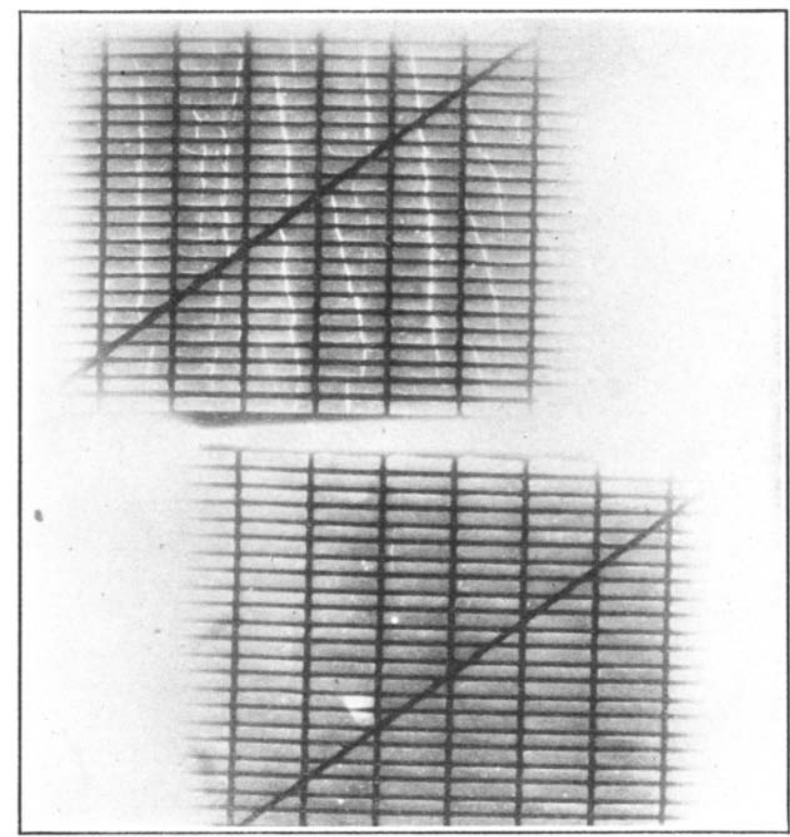

Twu storage battery grids. One has scveral cracks. Some of them not visible in ordinary light.

ing the amount of material acting, or else by using a clevice originally due to Bucky and recently developed by Potter.

The fluoroscopic and radiographic action depending on the unequal absorption of $\mathrm{X}$-rays at first found application only in medicine and surgery. The advent of the new tube capable of operation at high power for long periods without damage aroused interest in the radiography of various materials some examples of which may be mentioned here: Defects in small castings: grain of woods; defects in manufacture; pieces of metal in various objects. Fig. 6 shows a defective storage battery grid. 
Unfortunately both fluoroscopy and radiography have limitations of several kinds, the small thickness of dense materials radiable, time consumed, cost of apparatus, need for protection of operators, physical limits of vision in fluoroscopy, etc., but in many cases such examinations serve to point out defects due to improper processes or to errors readily avoided. Thus to examine hundreds of thousands of copper commutator bars would

FIG. 7 .

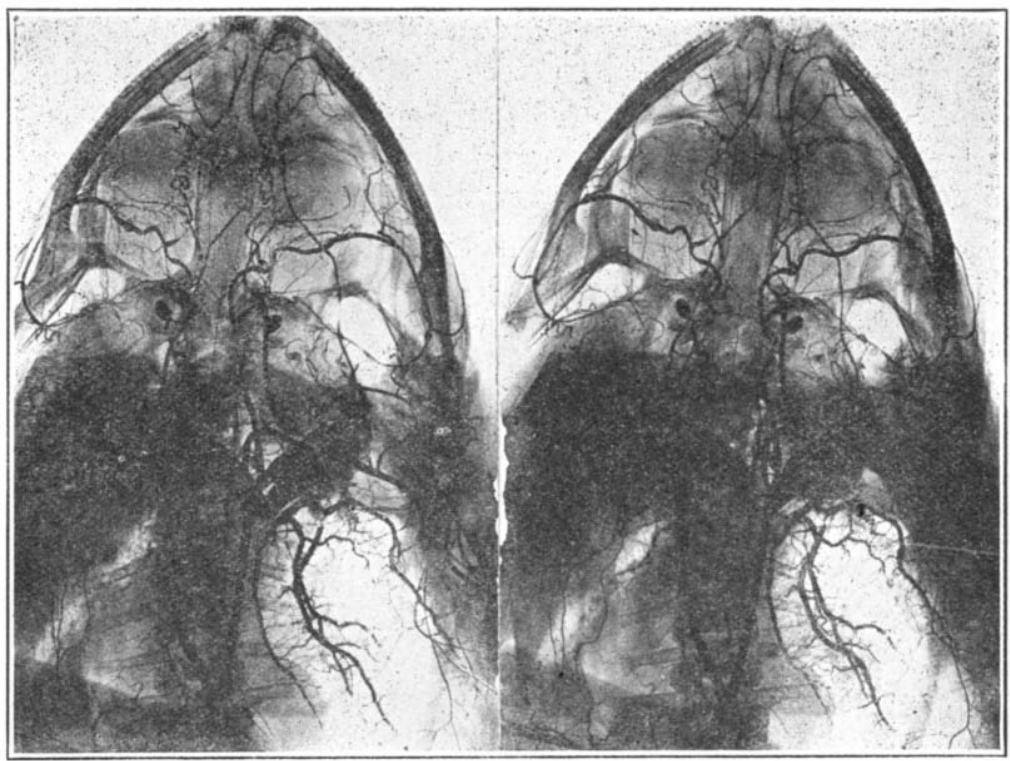

Circulatory (arterial) system of a frog. Shown by injecting the arteries and then radiographing.

be prohibitive, but to verify the elimination of gas by boronizing samples is relatively simple.

Where inspection can be made readily by fluoroscopy as in cork, mica, and some small objects, it may easily be made a routine procedure.

Our knowlerlge of animal and vegetable life and structure may be greatly increased by the use of this aid. Note circulatory system of a common amphibian secured by injection of a fluid carrying barium sulphate (Fig. 7).

Turning now to the second great advance that came in $19 \mathrm{I} 3$ we may consider briefly the phenomena of diffraction. The plane 
diffraction grating is cloubtless well known to most of you, but perhaps you have not considered the result of using several such gratings in succession on the same beam of light.

If $\lambda=$ wave-length

$\mathrm{d}=$ grating space.

$\frac{i}{d}=\sin \theta$

Since $\sin \theta$ ncver exceeds $\mathbf{I}$,

there is a minor limit to the wave-length for which a given grating may be used.

$\mathrm{d}=\frac{\lambda}{\operatorname{Sin} \theta}$, i.e., is always greater than $\lambda$.

But if $\theta$ is to be, say, 30 degrecs, $\sin \theta=\mathrm{J} / 2$

Then $\mathrm{d}=2 \lambda$, Suppose $\lambda=.00005, \mathrm{~d}=.000 \mathrm{I}=10^{-4}$ and we have need of 10,000 lines per $\mathrm{cm}$.

If $\lambda=10^{-8} \mathrm{~d}=2.10^{-6}$

No. lines per $\mathrm{cm} .=1 / 210^{6}=5 \times 10^{7}$

$$
=.50,000,000 \text { or fifty million lines per cm. }
$$

One would hardly try to rule as fine lines as this. But an entireiy different course of reasoning had shown that crystals were systematic atomic structures, where the atoms were arranged along straight lines with regular spacing. These again formed sets of parallel planes. The fundamental theory of the optical grating postulates the starting of exactly similar waves from each opening at the same instant or at regular time intervals. Laue conceived the idea that each atom might reflect a minute wave element and the regular arrangement of atoms might constitute a space grating for these very sloort wavelengths. A simple way of illustrating this will be to build for you a portion of a crystal enlarged in about the ratio of 50 milion to one. Thus you see the uniform spacing along lines ancl that parallel planes may be passed that contain regular atomic patterns (Fig. 8).

Many applications depend on this diffraction of crystals. For in this way we may ascertain two very important things: (I) The presence of elemetts by identification of their emission or absorption X-ray spectra, or (2) the presence and type of crystalline structure. The original Laue radiographs may serve in part for (2), but hardly for (1). Rellection methods may serve both ends. Thus the crystal X-ray spectrometer gave us a knowlelge 
FIG. 8.

(a)

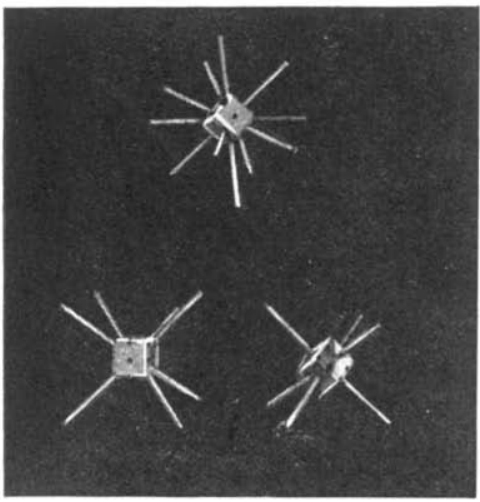

(1)

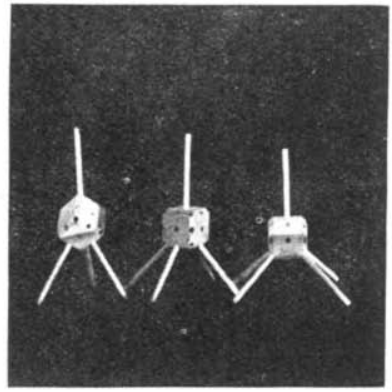

(c)

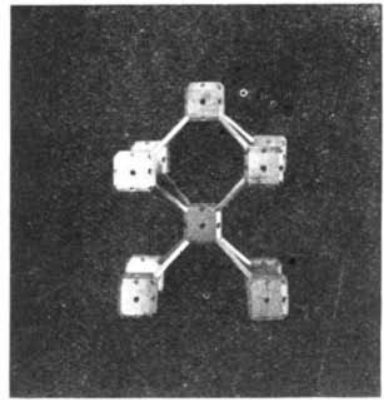

(d)

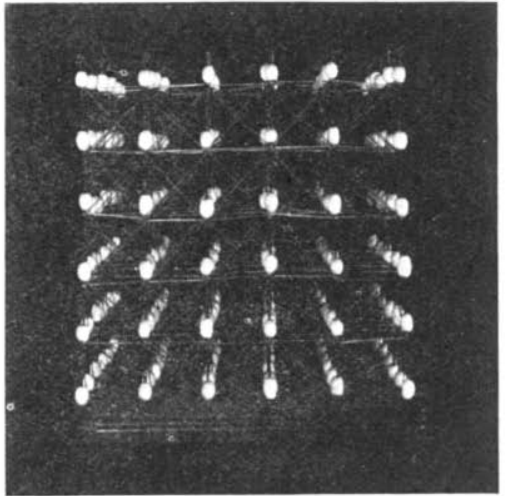

(e)

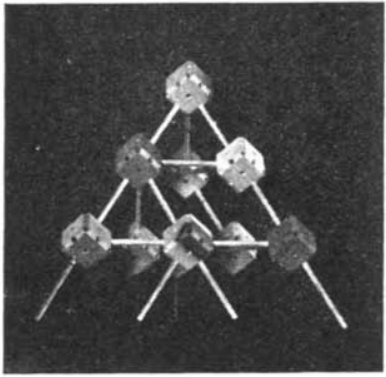

(f)

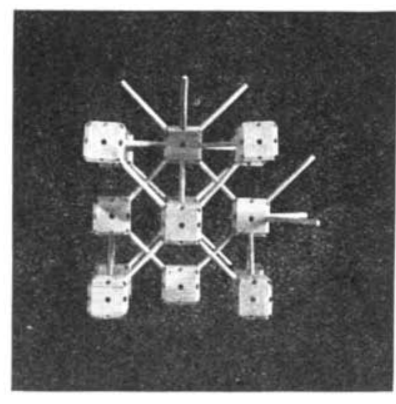

(a) Cubical blocks with holes and peys for use $\mathrm{n}$ building crystal models. Holes in the center of each face, the centcr of cach edge and cach corner. (b) The axial symmetries of such a system, four and three fold. (c) Part of a "cubic centered" structure. (d) Simple cubic. (c) Tetrahedral form of $f$. ( $f$ ) Part of a "face centered cubic" structure. 
of the X-ray spectrum and its variation with voltage, then, combined with other methods the rays verified the assumed nature of crystal structure, but even more they told just how the atoms were placed.

True the science of crystallography had been splendidly developed long before $\mathrm{X}$-rays were discovered, but so far as I am aware the exact position of various atoms, and the planes of highest concentration of a given type of atom had not been known. Also, this gives a method for the study of crystals of opaque substances such as metals in a manner not previously possible.

By usc of the crystal grating and an ionization chamber the FIG. 9.
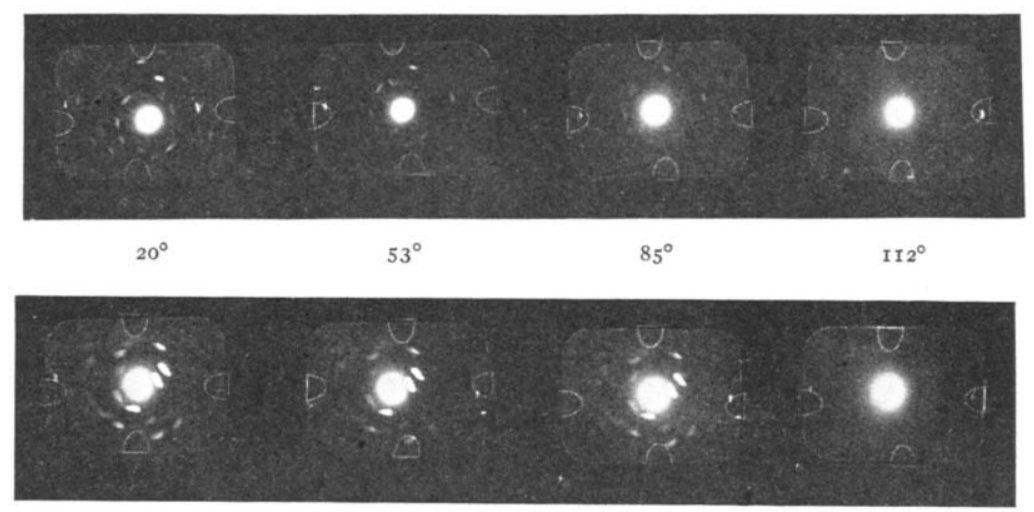

$58^{\circ} \mathrm{I} \mathrm{hr}$.

$62^{\circ} \mathrm{I} \mathrm{hr}$.

$62^{\circ} 2$ hrs.

$62^{\circ} 71 / 2 \mathrm{hrs}$.

Destruction of crystalline structure of copper sulphate by dehydration.

spectrum of $\mathrm{X}$-ray emission has been studied with reference to the wave-lengths of characteristic spectra and absorption and these results have been verified by photographic methods. An incandescent solid or liquid gives a continuous spectrum varying with the temperature.

A rise in temperature of a hot body adds short waves not present before; increases the intensity of radiation of all the wavelengths present at the lower temperature and shifts the energy peak toward the short wave-lengths. Also an incandescent gas emits a characteristic bright line spectrum that may be superimposed on a continuous spectrum from an incandescent liquid or solid. 
Change from temperature, as a controlling factor, to voltage, and we have the same laws in the radiation of X-rays. Further, the characteristic spectra of $\mathrm{X}$-ray emission are relatively very simple and are definitely related to the nature of the atom whose electrical equilibrium has been disturbed at the point of emission. In fact, if we arrange the elements in order by reference to these lines, we shall have with a few slight variations the order oi th ir atomic weight. Here again we break across those accidental boundaries of special sciences to invade the realm of the chemist. We no longer regard the atom as a simple lump of matter but rather as a complex electrical system.

The absorption spectra of X-rays are analogous to those of light. The coefficient of absorption increases with wave-length between two absorption bands. $\mu / \rho$ plotted against $\lambda^{3}$ gives a series of straight lines between bands due to the characteristic wave-lengths. Using these results it becomes possible to closely predict how the radiation described by a given spectrum will be distributed in an absorbing medium.

The entire history of an X-ray beam from its inception to its final complete degradation into heat is approximately shown by this chart drawn by Doctor Respondek. The career is surely very interesting and each step might offer a broad field for study.

I have not the time to dwell on that phase of X-ray application, the most widely known, and in sone ways the most important, suffice to say the possibilitics in the field of medicine are hardly realized to-day, great as we may consider present accomplishments.

Thus has a single epoch-making discovery wrought changes in our lives, our work, our thoughts. How many of the contemporaries of the patient investigators who contributed to the conditions rendering Röntgen's discovery possible realized the importance, or by material support or sympathy encouraged their humble efforts. Yet have these men greatly extended our knowledge with its possibilities for good or ill during a brief century. Sometimes they worked with a definite application in sight, more often were they inspired only by the desire to know more of the world in which man's sojourn is so brief.

That our science and philosophy has been and will still be profoundly modified by these results none will deny, so that these may say as the spirit in Faust,

"Thus at the ever whirring loom of time I ply,

And weave for Gor the garment that thou seest Him by." 